\title{
Pulling out the intentional structure of action: the relation between action processing and action production in infancy
}

\author{
Jessica A. Sommerville, ${ }^{\mathrm{a}, *}$, Amanda L. Woodward ${ }^{\mathrm{b}}$ \\ ${ }^{a}$ Department of Psychology, Institute for Learning and Brain Sciences, University of Washington, \\ Campus Box 357988, Seattle, WA 98195-7988, USA \\ ${ }^{\mathrm{b}}$ Department of Psychology, The University of Chicago, Chicago, IL, USA
}

Received 21 May 2003; revised 15 October 2003; accepted 29 December 2003

\begin{abstract}
Adults and children readily construct action representations organized with respect to an ultimate goal. These representations allow one to predict the consequences of action, interpret and describe actions, and categorize action sequences. In this paper, we explore the ontogeny of hierarchically organized action representations, and its relation to infants' ability to produce similar sequences. To do so, we examine infants' perception and performance of a means-end sequence: pulling a cloth to retrieve a toy. Using a visual habituation paradigm, we demonstrate that 12-month-old infants understand that the initial step of the cloth-pulling sequence is directed toward the ultimate goal of attaining the toy, and use their knowledge of the causal constraints of the sequence to make this goal attribution. Ten-month-olds, however, appear transitional with respect to this understanding: their ability to identify the goal of the cloth-pulling sequence is related to their own ability to planfully solve a similar sequence. These findings are consistent with a burgeoning body of literature suggesting an intimate link between action production and perception, and suggest that this link is in place by at least 10 months of age.

(C) 2004 Elsevier B.V. All rights reserved.
\end{abstract}

Keywords: Action processing; Action production; Intentional structure

\footnotetext{
* Corresponding author. Tel.: +1-206-616-6546.

E-mail address: sommej@u.washington.edu (J.A. Sommerville).
} 


\section{ARTCLEE TN PRESS}

\section{Introduction}

\subsection{Viewing action as hierarchically organized}

Adults extract the meaningful structure of action at multiple levels of analysis. For instance, we understand that reaching for a bag of flour indicates a desire to grasp the bag (the proximal goal), but we can also construe this action as one step among many directed toward achieving an ultimate goal: baking a cake. The ability to organize individual actions around ultimate goals is key to not only interpreting the actions of others, but also for predicting future actions based on past actions, for learning from and describing novel actions to others, and for categorizing action sequences in terms of the event representations to which they belong.

Philosophers and psychologists alike have contemplated our ability to construe actions at a basic level, as well as our ability to understand the relevance of such actions for realizing complex intentions. As discussed by Searle (p. 98, 1983), our construal of the murder of Archduke Franz Ferdinand in Sarajevo by Gavrilo Princip can exist at a number of different levels of analysis (e.g. pulling the trigger, firing the gun, shooting the Archduke, killing the Archduke, striking a blow against Serbia, avenging Serbia), each of which is embedded in the subsequent higher-level intention. Searle points out that we have the capacity to make intentional body movements where the conditions of satisfaction of our intentions go beyond the actual bodily movements produced, and that such an ability and appreciation is key to understanding meaning and causation. Indeed, as Searle writes, we can recognize that "Princip moved only his finger but his Intentionality covered the Austro-Hungarian Empire" (p. 99).

Theoretical work in psychology emphasizes the importance of construing events in a hierarchical manner. Problem solving (Newell \& Simon, 1972) and text comprehension (Schank \& Abelson, 1977) involve goal or plan identification and the organization of subgoals with respect to this plan. Event interpretation and description relies on our ability to conceive of everyday events as goal-directed partonomic hierarchies: we construe action events (such as making a bed) as composed of more basic action units (such as fluffing the pillows) (Newtson \& Engquist, 1976; Zacks et al., 2001; Zacks \& Tversky, 2001; Zacks, Tversky, \& Iyer, 2001).

Hierarchical action representations are also fundamental to a concept of intentions as independent of the particular actions that express them. Adults recognize that actions and goals do not bear an isomorphic relationship to one another, and therefore understand that a single action can have different meanings depending on the context in which it occurs. To return to our caking-baking example, the action of reaching for and grasping the handle of a scoop could be construed as directed toward obtaining the scoop itself (if it is sitting in a kitchen drawer), or as directed toward obtaining flour from the flour bag (if it is placed in the bag).

\subsection{The ontogeny of hierarchical action representations}

Given the centrality of viewing action as hierarchically organized in adult folk psychology, developmental theorists have considered it a critical step both for having 
intentions (Piaget, 1953) and for understanding intentions (Gergely, Nasady, Csibra, \& Biro, 1995; Meltzoff \& Moore, 1995; Tomasello, 1999). Indeed, the ability to construe action in terms of goals is not unique to adults. Preschoolers represent the behavior of others in terms of the goals that organize it (e.g. Bartsch \& Wellman, 1995; Slackman, Hudson, \& Fivush, 1986). Information about an actor's prior goals helps 2-year-olds imitatively learn a problem-solving task (Carpenter, Tomasello, \& Call, 2002), 18-monthold infants infer the goal of uncompleted action sequences (Meltzoff, 1995), and infants as young as 14 months imitate intended but not accidental acts (Carpenter, Akhtar, \& Tomasello, 1998).

Furthermore, recent work suggests that these goal-based action representations are hierarchically organized and reflect an ability to consider at least one aspect of the context, namely the causal relevance of an action to its goal. Infants reenact the final goal of a modeled action, but do not always reproduce the means (Gergely, Bekkering, \& Kiraly, 2002), suggesting that they recognize the final act as the ultimate goal that governs the sequence. Moreover, toddlers tendency to reenact or remember both entire action sequences (Wenner \& Bauer, 1999), and steps within a sequence (Travis, 1997) is influenced by the extent to which these steps bear causal relations to one another and to the goal of the sequence. These findings indicate that the origins of hierarchical action representations are to be found in infancy.

\subsection{Assessing action representations in young infants}

Recent findings indicate that two prerequisites for representing hierarchical goal structure are in place during the first year. First, by 6-9 months of age, infants represent certain single actions as directed at goals, rather than as purely physical trajectories through space (Jovanovic et al., under review; Woodward, 1998, 1999, 2003). To illustrate, when infants were habituated to an event in which a person grasped an object, they subsequently demonstrated a strong novelty response to events which maintained the physical properties of the reach but disrupted the relation between the person and her goal, but showed no such response to events which varied the physical properties of the reach while maintaining the goal (Woodward, 1998). Second, infants seem able to flexibly interpret the same observed motion as goal-directed or not depending on the causal context. To illustrate, in one study (Gergely et al., 1995), 12-month-old infants viewed a computer animation in which a figure moved across the screen along a circuitous path, ultimately reaching another figure at the far side of the screen. When the path circumvented a barrier, infants responded to the motion as if it were goal-directed. When there was no barrier present, infants did not respond in this way. Several studies have documented this kind of flexible action interpretation in infants from 9 to 12 months of age (Csibra, Biro, Koos, \& Gergely, 2003; Csibra, Gergely, Biro, Koos, \& Brockbank, 1999; Gergely et al., 1995; Wellman \& Phillips, 2001), and other findings suggest that even younger infants may show this ability (Jovanovic et al., under review; Kiraly, Jovanovic, Prinz, Aschersleben, \& Gergely, 2003; but see Heineman-Pieper \& Woodward, in press).

In a recent study (Woodward \& Sommerville, 2000), we tested whether beyond these two starting abilities, infants are able to represent the hierarchical goal structure of an action sequence. That is, we asked whether infants can interpret an action with a clear 


\section{ARTICLE TN PRESS}

proximate goal (grasping) as directed not at the object acted upon, but, instead, at the ultimate goal of the action sequence in which it was embedded. Twelve-month-old infants saw two colored translucent boxes sitting side by side on a stage, each of which contained a different toy. During the habituation portion of the study, an actor reached in, grasped the lid of one of the boxes and then opened the box and grasped the toy. Then the locations of the toys were switched and infants saw only the first action of the sequence (the touch to the box lid) during test trials. On half of the test trials (new goal trials), the actor grasped the same box lid that she had initially, now containing a new toy. On the other half of the test trials (new means trials) the actor grasped the lid of a different box than she had initially, which contained the same toy that she had grasped during habituation trials. If infants construed the touch to the box lid as directed toward the toy they should show longer looking on the new goal trials. In contrast, if infants viewed the actor's touch to the box lid as directed toward the lid itself (the proximal goal, or means, of the sequence) they should look longer on the new means trials.

Infants showed the former pattern, suggesting that they recognized the touch to the box lid as a means to attaining the toy. A follow-up study confirmed that 12-month-olds used their causal knowledge to construct their action representations. When shown a similar sequence in which the toy sat outside of the box, infants did not view the actor's touch to the box lid as directed toward the attainment of the toy. These findings indicate that 12-month-old infants interpret single actions within a sequence as directed toward a higher-order goal, and that infants link actions to higher-order goals based on their causal role in goal attainment.

\subsection{Developmental contributions to constructing action representations}

There is currently a diversity of opinion concerning the extent to which infants' action representations derive from experience versus innately specified conceptual content (see e.g. Csibra et al., 1999; Kiraly et al., 2003; Woodward, Guajardo, \& Sommerville, 2001). Nevertheless, regardless of the theoretical perspective from which early action understanding is approached, there is a general consensus that infants' action representations are enriched and elaborated over the first year of life (e.g. Csibra et al., 2003; Gopnik \& Meltzoff, 1997; Woodward et al., 2001). The question of interest concerns the knowledge and abilities that contribute to this development.

\subsubsection{Domain-general and domain-specific contributions}

Infants' ability to parse actions (e.g. Baird \& Baldwin, 2001), integrate information across objects and events (e.g. Cohen, 1998; Cohen, Chaput, \& Cashon, 2002), and to inhibit and temporally sequence actions and representations (Diamond, 1991) are domaingeneral intellectual capacities that are developing over the first year of life which may be integral to infants' ability to create structured action representations centered around an overarching goal. Event-specific information may also contribute to the development of structured action representations. For instance, adult action understanding is informed by rich knowledge of the social and psychological constraints that govern goal attribution. Although such knowledge may be limited in infants, work on infant action representations suggests that infants readily consider the causal constraints that govern 
action (see Section 1.2), suggesting that infants' developing ability to reason about particular causal situations may contribute to their hierarchical action representations (cf. Csibra et al., 2003, 1999; Gergely et al., 1995).

\subsubsection{Observational and agentive experience}

Piaget (1953) was one of the first to suggest that early action experience preceded and contributed to later representational understanding. Beyond a general effect of action experience on cognition, observational and agentive experience may help infants learn about the goal-directed nature of action sequences by providing specific information about particular actions. Through watching others, infants may learn about the action outcomes associated with a variety of acts (e.g. Jovanovic et al., under review) that highlight the goal structure of actions. In turn, the detection of these action goals may influence infants' subsequent action planning and control (Hauf, Elsner, \& Aschersleben, in press).

Infants may also glean important information about goal-directed actions as a result of their own self-produced actions. Indeed, the information gained through self-experience may be particularly rich because not only does it provide infants with more exemplars of goal-directed action, but it also provides them with information that is uniquely specified when the self is acting (such as information about internal states).

Philosophers have speculated that the self, and self-actions in particular, play a foundational role in understanding others, both in terms of the development of action understanding (e.g. Baldwin, 1897; Cooley, 1902) and with respect to our ability to understand the behavior of others on-line (e.g. Goldman, 1989; Gordon, 1986; Harris, 1989; Heal, 1989). In addition, developmentalists have suggested that action production and understanding are deeply intertwined and that this relation may serve as a powerful engine in the development of social understanding (Meltzoff, 2002b; Meltzoff \& Brooks, 2001; Tomasello, 1999; Woodward et al., 2001). Recently the idea that we use selfexperience to interpret other experience (or vice versa) has also been advocated by neuroscientists based on evidence of an observation/execution matching system in humans. Some have suggested that this system may be part of, or a precursor to, our ability to interpret others' actions with respect to their underlying intentions or other mental states (e.g. Blakemore \& Decety, 2001; Gallese \& Goldman, 1998; Meltzoff \& Decety, 2003; Rizzolatti, Fadiga, \& Gallese, 2001).

Because action processing and action production may be affected by similar domaingeneral processes, and because observational and agentive experience may yield important information about the structure of goal-directed action and guide action control, it is possible that action processing and production are related, particularly early in development. To date, very few studies have examined the relation between habituation task performance and performance across other tasks purported to measure similar types of knowledge, although some that have indicate dissociations in infants' physical reasoning abilities in action and perception (e.g. Hood, Cole-Davies, \& Dias, 2003). As such, a demonstration assessing the potential relation between action processing and production in infancy would provide important information regarding the accessibility of knowledge measured in habituation tasks to other modalities, contribute to knowledge on the experiential dependence of developing action representations in infancy, and add to 


\section{ARTICLE TN PRESS}

the growing body of literature suggesting a shared basis for the processing and production of action.

\subsection{Overview of the current studies}

The studies in this paper examine infants' ability to construct hierarchically organized action representations (Study 1) and the relation between action production and action processing in infancy (Study 2). To preview, the findings from Study 1 suggest that infants have a basic ability to construct hierarchical action representations by the end of the first year of life, and that this ability is informed by infants' knowledge of the causal context in which action is occurring.

In Study 2 we investigated the emergence of infants' understanding of hierarchical action sequences. Our findings suggest that 10-12 months of age represents an important transitional period in infants' action understanding. Between these ages there are developments in infants' ability to construct hierarchical representations of action sequences and to perform these sequences in a planful manner. These findings also shed light on the relation between infants' developing action abilities and their action interpretations.

\section{Study 1}

In Study 1, we sought converging evidence for the findings of Woodward and Sommerville (2000). To date, these findings are the only demonstration that infants can construe a familiar object-directed action in terms of its relation to other actions in a means-end sequence. We showed infants events in which a person pulled a cloth toward herself in order to obtain a toy it supported. We chose this sequence because this task has proven to be a fruitful measure of infants' own means-ends abilities and a number of researchers have pointed out that infants can solve this sequence by 12 months of age, if not several months earlier (Munakata, McClelland, Johnson, \& Siegler, 1997; Piaget, 1953; Willatts, 1990, 1999). As such, the cloth-pulling sequence seemed to be amenable for both exploring 12-month-olds' structured action representations and for investigating the developmental origins of this ability.

The question of interest in this study was whether infants could construe the act of cloth pulling as directed toward the ultimate goal of the sequence: attaining the toy. To pose this question, we used a visual habituation paradigm. Infants watched live events that were presented on a small stage. During the habituation phase, infants saw an actor pull a cloth that supported a distant toy and then grasp the toy. After habituation, infants saw test events in which the actor acted only on the cloth. In some test events the actor acted toward a new ultimate goal using the same means that she had during habituation (new goal trials). In other test events the actor acted toward the same ultimate goal that she had during habituation, now using a different means (new means trials). We predicted that if infants construed the initial completed cloth-pulling sequence as directed toward the ultimate goal of attaining the toy they would look longer at new goal trials than at new means trials. In contrast, if infants construed the cloth grasp only in terms of the means (grasping 
the cloth), and not the ultimate goal (attaining the toy), we would predict that they would look longer on new means trials than on new goal trials.

To ensure that infants considered the causal constraints of the sequence when making their goal attributions, another group of infants were tested in a control condition in which the toy sat adjacent to the cloth rather than on it. In this condition, the temporal correspondence between cloth pulling and toy grasping was retained, but the causal relation (necessary for adults to construe the cloth-grasp as directed toward attaining the toy) was disrupted. If infants took into account the causal relation between pulling the cloth and grasping the toy in assigning their goal interpretations, then it was predicted that infants in this condition would show no significant preference for either type of test event.

\subsection{Method}

\subsubsection{Participants}

Thirty-two 12-month-old infants participated in the experiment. Infants ranged in age from 10 months, 25 days to 13 months, 1 day (mean age $=11$ months, 27 days). Seventeen of the infants were female and 15 were male. The infants were all full term (at least 37 weeks gestation) and from a large city in the United States. Parents volunteered to participate in response to advertisements and mailings. Ten additional infants began the procedure but were not included in the final sample because they became fussy during the task $(n=1)$, there was an experimental error $(n=5)$, or because their gaze was not visible on the camera for part of the procedure $(n=4)$. Infants were randomly assigned to either the toy-on-cloth condition (mean age $=11$ months, 26 days), or the toy-off-cloth condition (mean age $=11$ months, 28 days).

\subsubsection{Materials and procedure}

The events were presented on a stage $(76 \mathrm{~cm} \times 86 \mathrm{~cm} \times 152 \mathrm{~cm})$, which consisted of an area surrounded by black cloth on three sides. A table was situated in front of the stage $(79 \mathrm{~cm} \times 23 \mathrm{~cm} \times 86 \mathrm{~cm})$. Infants were seated on the table, in either the parent's lap or in an infant seat facing the stage (approximately $69 \mathrm{~cm}$ from the display). A white screen could be raised manually to block the infants' view of the stage.

The stage floor contained two cloths ${ }^{1}$ each of which supported a different toy (see Fig. 1a and b). Both of the cloths were mounted on a slightly elevated black platform. This allowed for each cloth to be attached to the platform with hidden screws so that the cloth would move in a track when pulled. The cloths, one blue and one yellow, each measured $20 \mathrm{~cm} \times 33 \mathrm{~cm}$. The toys each measured $5 \mathrm{~cm} \times 6.5 \mathrm{~cm}$. One was a green plastic frog and one was a red plastic fish. In the toy-on-cloth condition, each toy sat on one of the cloths, $7.5 \mathrm{~cm}$ from its leading edge. In the toy-off-cloth condition, each toy sat adjacent to one of the cloths, $7.5 \mathrm{~cm}$ from its outer edge. An actor sat between the two cloths, on the other side of the platform such that she was facing the infants when the screen was lowered. A video camcorder was mounted at the rear of the stage to capture the infants' eye gaze. The camera was hidden by a black curtain with only its lens protruding.

\footnotetext{
${ }^{1}$ The cloths were actually thin, rectangular pieces of cardboard covered with colored paper.
} 


\section{ARTICLE IN PRESS}

2.1.2.1. Habituation trials. Fig. 1a and b illustrates the habituation and test trials for the toy-on-cloth and toy-off-cloth conditions, respectively.

The habituation phase began when the white screen was lowered to reveal the display. On each trial, infants viewed an actor sitting between the two cloths, across from the infant. The actor said "Hi. Look", and reached toward and grasped the outer edge of one of the cloths, pulled it toward her, and grasped the toy sitting on the cloth. For half of the infants, the actor pulled the cloth on the infants' left (the yellow cloth) and for half of the infants the actor pulled the cloth on the infants' right (the blue cloth). Recording of infants' looking began once the actor had grasped the toy and ended when the infant looked away from the event for two consecutive seconds. At the end of each trial, the screen was raised and then lowered to begin the next trial.

Habituation event

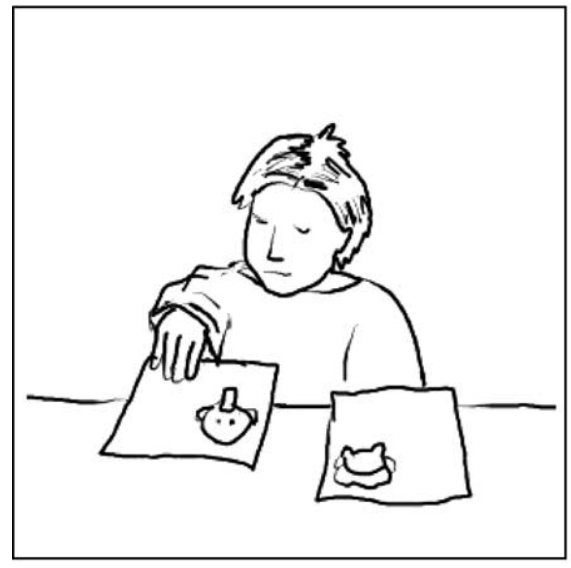

New goal event

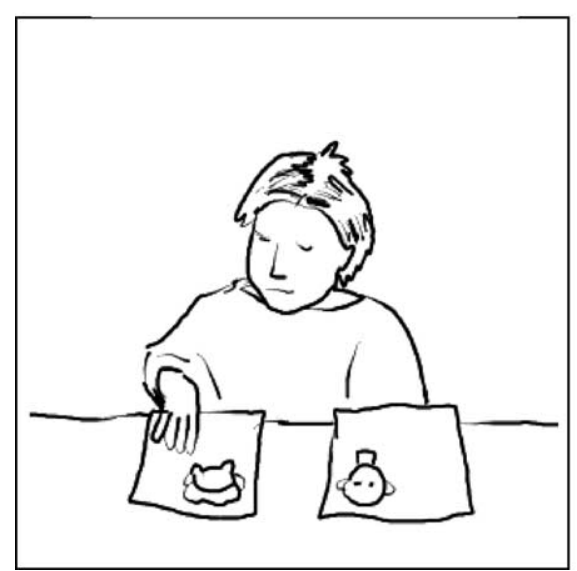

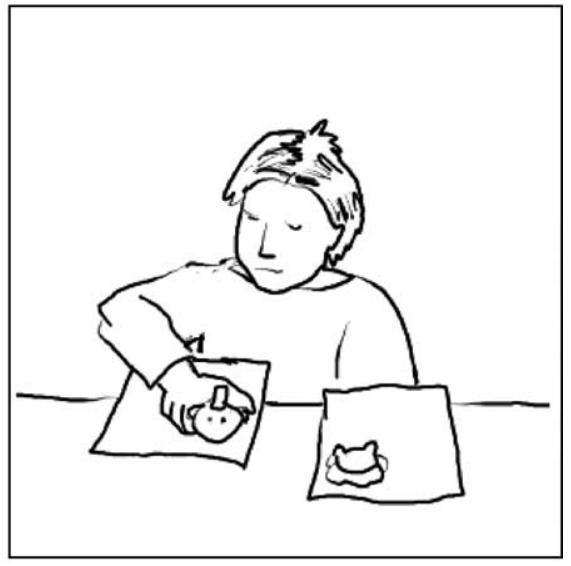

New means event

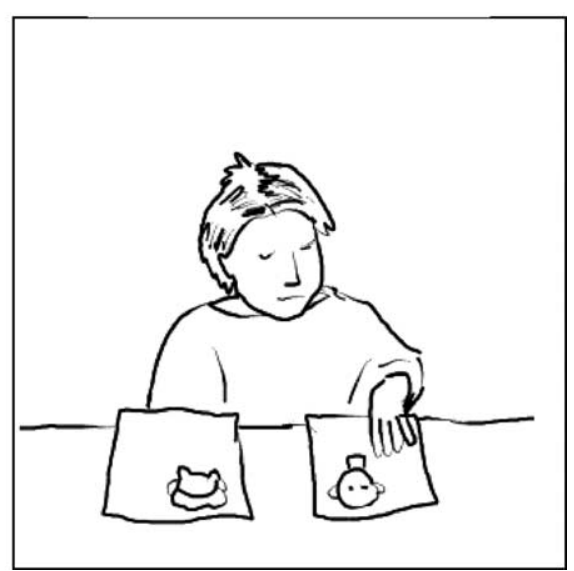

Fig. 1. (a) Toy-on-cloth condition. (b) Toy-off-cloth condition. 
Habituation event

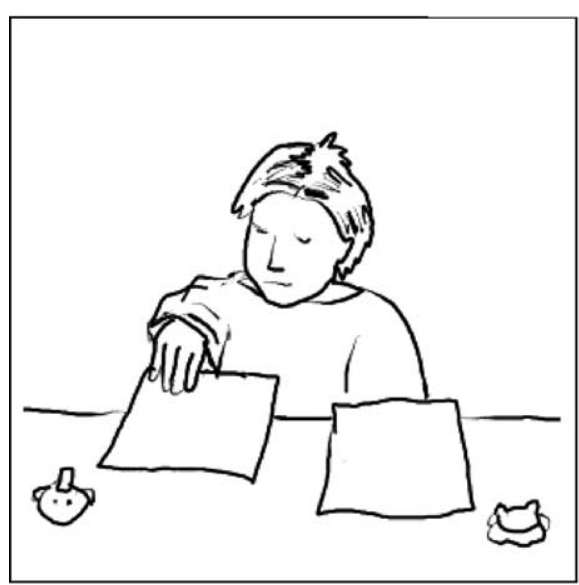

New goal event

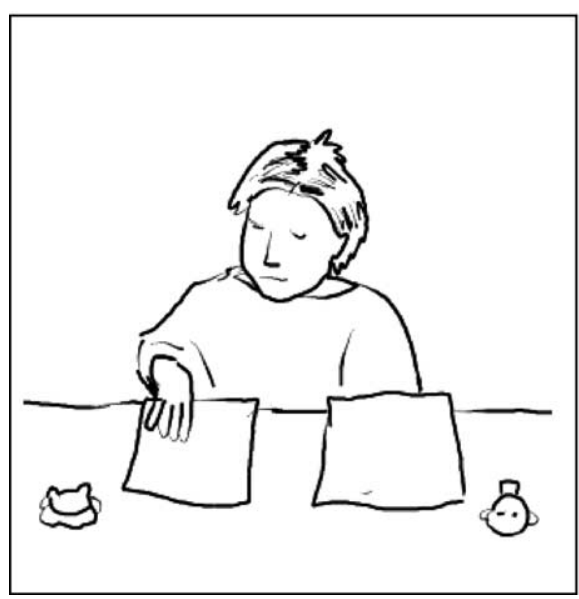

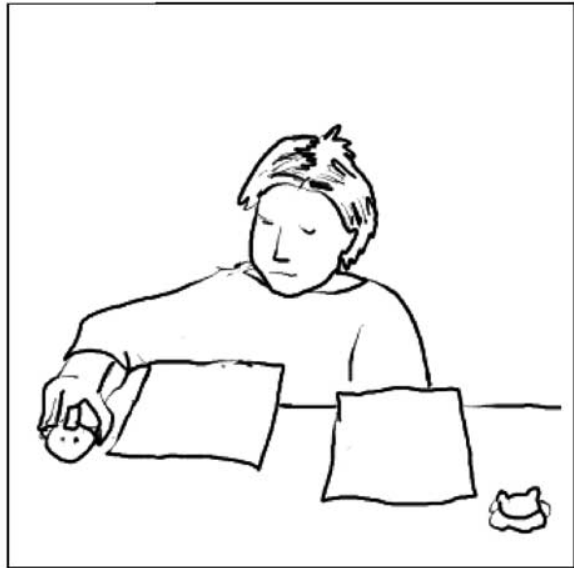

New means event

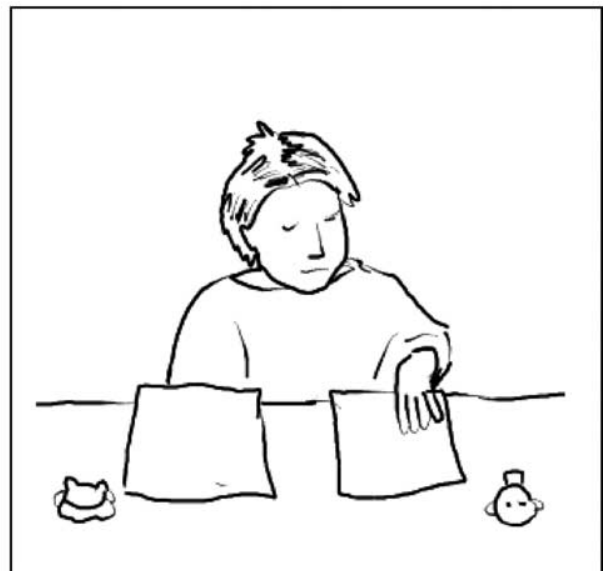

Fig. 1 (continued)

The habituation phase ended after infants' looking time, totaled across three consecutive trials, fell to half of its initial level on the first three habituation trials. Thus, each infant received a minimum of six habituation trials. If the infant had not met the habituation criterion after 14 trials, the habituation phase was ended and test trials began.

2.1.2.2. Test trials. Prior to the test phase the screen was raised and the locations of the toys were switched; the cloths stayed in place. On test trials the actor said "Hi. Look", then grasped one of the cloths and remained still. Infants' looking was recorded from the time that the actor grasped the cloth, until the infant looked away from the event for two 


\section{ARTCLEE TN PRESS}

consecutive seconds. On new goal trials, the actor grasped the same cloth she had grasped during habituation, which now supported (toy-on-cloth condition) or was adjacent to (toyoff-cloth condition) a different toy. On new means trials, the actor grasped the other cloth that supported (toy-on-cloth condition) or was adjacent to (toy-off-cloth condition) the toy that had been acted on during habituation trials. Each infant received three trials of each type in alternation for a total of six test trials. Half of the infants in each condition received the new goal event first, and half received the new means event first.

2.1.2.3. Coding. Infants' looking time was calculated on-line by an observer who watched the infant on a video monitor. The observer pushed a computer key when the infant was looking at the area encompassing the cloths, toys and the actor. Looking time was calculated from the time the actor stopped moving (e.g. grasped the toy on habituation trials, or grasped the cloth on test trials) until the infant looked away for $2 \mathrm{~s}$. The observer was unaware of trial type and condition and was able to see only the infant on the monitor. A computer program was used to calculate looking times and the habituation criteria (Pinto, 1994).

2.1.2.4. Reliability. A secondary observer, who was unaware of the trial type as well as to the condition that the infants participated in, coded the infants' gaze from videotape. Observers were counted as agreeing if they identified the same look away as ending the trial. The primary and secondary observers agreed on the ending of $92 \%$ of test trials for the toy-on-cloth condition and $90 \%$ of test trials for the toy-off-cloth condition. To ensure that disagreements did not occur systematically in favor of the hypothesis the disagreements were categorized into two groups: those that would have contributed to the hypothesized pattern of findings and those that would have worked against the hypothesized pattern of findings. Disagreements were randomly distributed across these categories for both the toy-on-cloth-condition $\left(X^{2}(\mathrm{df}=1)=0.03, P>0.86\right)$ and the toyoff-cloth condition $\left(X^{2}(\mathrm{df}=1)=0.90, P>0.34\right)$.

\subsection{Results}

Fig. 2 summarizes infants' looking times for the habituation and test trials for both the toy-on-cloth and toy-off-cloth conditions.

\subsubsection{Habituation}

Infants in the two conditions showed different levels of attention in the habituation phase. Looking times on the last three habituation trials differed as a function of condition $(t(31)=2.00, P<0.05)$. Infants' looking times in the toy-on-cloth condition averaged 16.0 $(\mathrm{SE}=2.4) \mathrm{s}$ on the last three habituation trials and infants' looking times in the toy-off-cloth condition averaged $10.5(\mathrm{SE}=1.4) \mathrm{s}$. These findings mirror those of other studies that report that infants pay less attention to actions that are not wellorganized with respect to a goal (Csibra et al., 1999; Wellman \& Phillips, 2001). Infants' rate of habituation, however, did not differ as a function of condition. Infants in the toyon-cloth condition averaged $8.3(\mathrm{SE}=2.8)$ habituation trials and infants in the toy-offcloth condition averaged 8.1 ( $\mathrm{SE}=2.3)$ habituation trials $(t<1.0)$. Two infants in 


\section{ARTICLE TN PRESS}

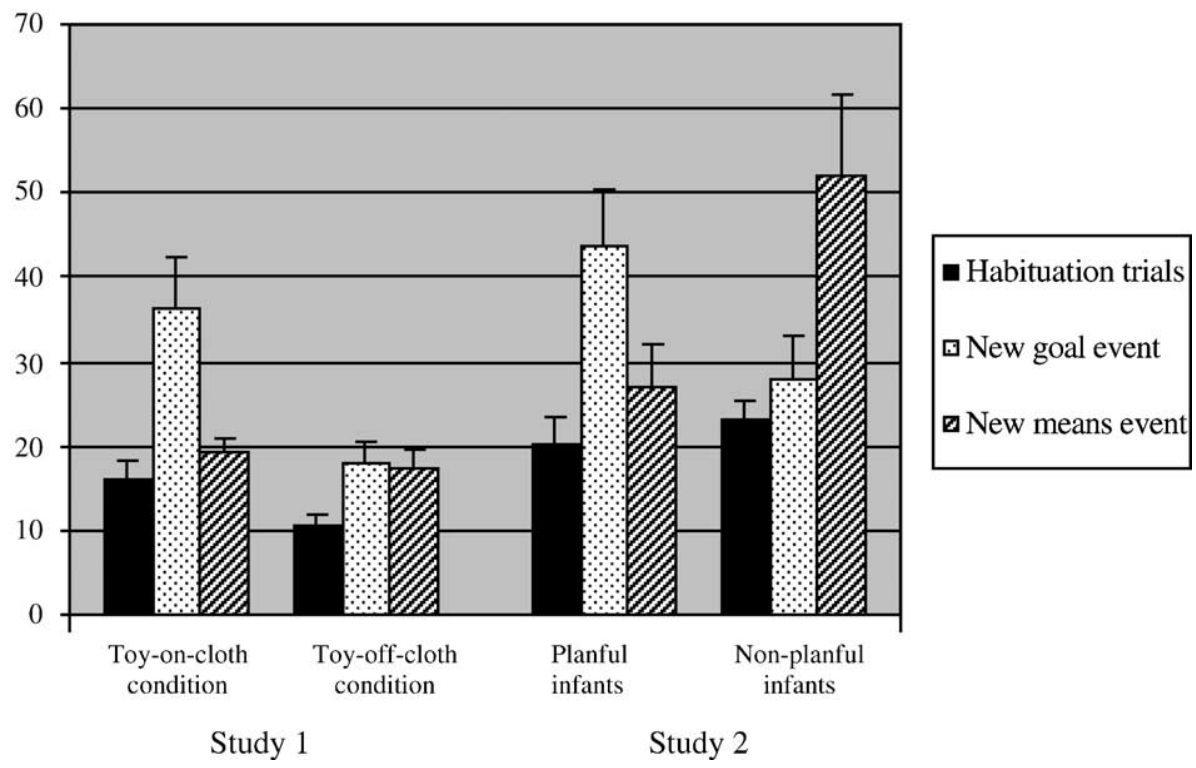

Fig. 2. Mean looking times to the last three habituation trials and test events, Studies 1 and 2.

the toy-on-cloth condition and one infant in the toy-off cloth condition completed 14 habituation trials without reaching the habituation criterion. ${ }^{2}$

\subsubsection{Test}

The primary analyses assessed infants' attention to new goal versus new means test events. Preliminary analyses indicated no reliable effects of the side to which the actor first reached, the toy that was reached for, or the test trial type given first on looking times to the test events. Therefore, we collapsed across these factors in subsequent analyses. Moreover, preliminary analyses indicated that infants' looking to the two test trial types did not vary as a function of trial pair. Therefore, infants' total looking times across the three trials of each type were entered into the main analyses.

We entered these scores into an ANOVA with condition (toy-on-cloth versus toy-offcloth) as the between-subjects variable, and test event (new goal versus new means event) as the within-subjects variable. This analysis revealed a main effect of condition $(F(1,30)=5.90, P<0.02)$, reflecting the fact that infants in the toy-on-cloth condition looked longer overall to the test events $(M=71.5, \mathrm{SE}=8.3)$ than did infants in the toyoff-cloth condition $(M=47.1, \mathrm{SE}=5.1)$. In addition, this analysis revealed a main effect of trial type $(F(1,30)=4.76, P<0.05)$, reflecting the fact that looking on the test trials was longer to the new goal event $(M=26.9, \mathrm{SE}=3.6)$ than the new means event $(M=19.2, \mathrm{SE}=1.8)$. These main effects were qualified by a trial type by condition interaction $(F(1,30)=7.29, P<0.01)$. To resolve this interaction, we next performed

\footnotetext{
${ }^{2}$ Data analyses were completed for all infants and for only those infants who reached the habituation criteria. Because the exclusion of the data from those infants who failed to habituate did not change the pattern of findings, we chose to retain their data in the final analyses.
} 


\section{ARTICLE TN PRESS}

planned comparisons on looking time to the test events for each condition. These analyses revealed that infants in the toy-on-cloth condition looked longer on the new goal trials than on the new means trials $(t(15)=2.90, P<0.01)$, but infants in the toy-off-cloth condition showed no significant preference for either type of test event $(t(15)=1.83, P>0.63)$. Performance at the individual level mirrored these findings. Thirteen of 16 infants in the toy-on-cloth condition looked longer to the new goal event than new means event $(P<0.02$, paired sign test). In contrast, only half of the infants in the toy-off-cloth condition $(n=8)$ looked longer at the new goal test event $(P>0.99)$.

Our final analyses focused on infants' recovery to the test events from habituation. This analysis is important because it is possible that infants in the toy-off-cloth condition failed to differentiate between the new goal and new means events due to lack of overall attention, thus accounting for the difference in looking patterns across the two conditions. Infants' looking times on the last three habituation trials was compared to their looking times on the three new goal test trials and the three new means test trials. ${ }^{3}$ Paired $t$-tests revealed that infants in the toy-off-cloth condition reliably recovered attention to both types of test events $(t(15)=2.13, P<0.05$ for the new goal event and $t(15)=3.00$, $P<0.01$ for the new means event), indicating that infants in this condition responded to both types of changes. Infants in the toy-on-cloth condition reliably recovered attention to the new goal event $(t(15)=4.66, P<0.01)$, but not the new means event $(t(15)=1.14$, $P>0.27$ ). These findings suggest that infants in the toy-on-cloth condition discriminated between the habituation event and the new goal event, but did not differentiate between the habituation event and the new means event.

These findings are similar those of Woodward $(1998,1999,2003)$. Specifically, infants across these studies appear to selectively recover attention to changes in the goal-relevant aspects of actions that adults interpret as goal-directed, and to recover attention to both changes in the goal-relevant features and the spatial/perceptual features for actions that are not interpreted as goal-directed by adults.

\subsection{Discussion}

The results from Study 1 suggest that 12-month-old infants understand the cloth-pulling sequence as hierarchically organized. After watching a completed action sequence in which an actor pulled a cloth to obtain a toy, infants understood that the actor's subsequent actions on only the cloth were directed toward the toy (and not the cloth itself). ${ }^{4}$

\footnotetext{
${ }^{3}$ Recovery analyses can be difficult to interpret since habituation trials are artificially selected for comparison to test trials based on low levels of looking, and, as such, "recovery" may be artificially created on the basis of this selection. However, we point out that in the case of differential recovery to test events, this objection is of less concern.

${ }^{4}$ It is unlikely that experimenter bias could not account for infants' differential looking on the test trials as infants' looking was timed to the static outcome of the events. However, to further ensure that this was not the case, a subset of new goal and new means test events (25\%) from the toy-on-cloth and toy-off-cloth conditions of Study 1 were coded by an observer who was naïve to the purposes of the study to determine whether this observer could identify presentation biases in favor of the hypotheses. Judgments were categorized according to whether the observer predicted the infant would look longer at the new goal vs. new means event for both the toy-on-cloth and toy-off-cloth conditions. This analysis revealed that the observer's predictions were randomly distributed for both conditions $\left(X^{2}(\mathrm{df}=1)=0.69, P>0.41\right)$.
} 
Moreover, the results from the toy-off-cloth condition provide evidence that this understanding is not based solely on the temporal contiguity of the actions within the sequence. When the causal relation between pulling the cloth and grasping the toy was disrupted infants did not interpret the actor's touch of the cloth as directed toward the toy.

Another explanation for our findings could be that infants identified the goal of the habituation sequence in the toy-on-cloth condition by using a simple perceptual rule. One way in which the toy-on-cloth and toy-off-cloth conditions differ from one another is that whereas the toy undergoes motion in the toy-on-cloth habituation event, it does not in the toy-off-cloth habituation event. Thus, infants may have demonstrated a new goal event preference for the test events in the former condition, but not the latter, because they focused on the movement of the toy and used only this information to identify the actor's goal (e.g. "the moving toy is the goal"). To ensure that infants' novelty preference for the new goal event in the toy-on-cloth condition was not due to this simple motion rule, we tested another group of 16 12-month-old infants in the toy-moves condition. This condition was identical to that of the toy-off-cloth condition, except that after grasping the toy on the habituation trials, the actor moved it several inches toward her. Infants' looking times to the test events in this condition did not differ significantly $(t(15)=0.72$, $P>0.8$ ), suggesting that infants' new goal preference in toy-on-cloth condition was not due to the construction of this simple perceptual rule.

Although infants looked less overall at the events in the toy-off-cloth condition, the results from the recovery analyses suggest that the failure to find a difference between the two conditions is not a result of low levels of attention by the infants in the toy-off-cloth condition. Indeed, the fact that infants in the toy-off-cloth condition demonstrated shorter overall looking times to the habituation and test events than did infants in the toy-on-cloth condition is in keeping with findings from other studies (e.g. Wellman \& Phillips, 2001) suggesting that infants may find actions that are not clearly goal-directed less interesting than goal-directed actions.

Taken together with the findings of Woodward and Sommerville (2000), our results suggest that infants may be able to interpret a range of simple action sequences as directed toward an ultimate goal by the end of the first year of life (although infants' ability to do so may be influenced by various task-specific variables, such as the extent of similarity between the sub-goal and goal action). Moreover, our findings suggest that infants use some of the same parameters in processing action sequences as do adults. Infants consider the causal relations between actions when linking them within a sequence, and use these relations to specify the ultimate goal of the action sequence. As such infants are not merely associating actions within a sequence based upon their temporal continuity, but rather use top-down knowledge to influence the extent to which they rely on statistical regularity in their action processing.

\section{Study 2}

In Study 2 we investigated the developmental origins of the ability to view action sequences as hierarchically organized. We did this for two reasons. First, the presence or absence of the ability to understand action sequences as directed toward an ultimate goal in 


\section{ARTICLE IN PRESS}

younger infants has implications for whether this ability is a developmental achievement or an innate endowment. Second, information regarding the developmental time course of this aspect of action processing can nominate candidate mechanisms that may be responsible for changes in infants' construction of action representations. In Study 2 we explored one capacity that may be integrally linked to infants' action understanding: infants' own developing ability to solve similar means-end sequences.

Work on infants' ability to solve simple means-end sequences (such as reaching around a barrier to retrieve a toy, reaching into a box to obtain an object, and pulling a cloth to grasp an out-of-reach toy) suggests that this form of problem solving follows a protracted development, but that 9-12 months of age marks a particularly important developmental period in infants' ability to spontaneously solve one-step means-end sequences in a planful manner (e.g. Bates, Carlson-Luden, \& Bretherton, 1980; Diamond, 1985; Piaget, 1953; Uzgiris \& Hunt, 1975; Willatts, 1990). Starting at 6 or 7 months of age infants can be trained to act on one object in order to attain another (Menard \& Aguiar, 2002; Munakata et al., 1997). Over the next several months, infants' behavior on means-end tasks becomes increasingly systematic and spontaneous (e.g. Willatts, 1999). Infants' success on means-ends tasks depends on several factors including the ease with which the intermediary and goal appear visually distinct, and the amount of spatial contact between the goal and intermediary (Bates et al., 1980), and infants' ability to correctly select a causally effective tool continues to improve through the second year of life (e.g. Chen \& Siegler, 2000).

There are a number of theoretical reasons to expect a relation between production and comprehension of means-ends actions. Between 9 and 12 months of age infants are developing a range of general-purpose abilities that may govern performance across a variety of tasks (e.g. Piaget, 1953). Changes in information processing, the development of a general means-end system or improvements in inhibitory control may have functional consequences for both action production and interpretation. In contrast, action processing and production may be directly linked because information about the goal-structure of action gained from observing and experiencing one's own actions may be applied to infants' interpretation of others' acts or may help improve infants' action control.

In Study 2, infants' developing ability to understand the hierarchical nature of action sequences was examined by testing 10-month-olds in a habituation task and a similar action task. The habituation task tapped infants' construals of the cloth-pulling sequence as performed by another person and was identical to that of the toy-on-cloth condition from Study 1 . The action task tapped infants' own ability to solve the sequence. Because previous work (Willatts, 1990) suggests that means-end sequences, such as cloth-pulling sequences, may be solved fortuitously, the extent to which infants solved the cloth-pulling task in an apparently intentional manner was taken into account. In order to examine the relation between action task performance and habituation task performance, half of the infants participated in the action task prior to the habituation task (action first group), and half of the infants completed the tasks in the reverse order (habituation first group). The results are analyzed both with respect to the effect of performing the cloth-pulling sequence directly prior to viewing another person perform the same sequence, and the relation between individual performance on the habituation task and the action task. 


\section{ARTICLEE IN PRESS}

\subsection{Method}

\subsubsection{Participants}

Forty-eight 10-month-old infants participated in the experiment. Infants ranged in age from 8 months, 29 days to 10 months, 29 days (mean age $=9$ months, 19 days). All infants participated in a habituation task and an action task. Half of the infants were assigned to the action first group (receiving the action task first) and half were assigned to the habituation first group (receiving the habituation task first). Twelve of the infants in the action first group were male and 12 were female. Infants in this group ranged in age from 8 months, 29 days to 10 months, 18 days (mean age $=9$ months, 20 days). Seven of the infants in the habituation first group were male and 17 were female. Infants in the habituation first group ranged in age from 9 months, 0 days to 10 months, 29 days (mean age $=9$ months, 20 days). The recruitment procedures and testing location was identical to that of Study 1. Six additional infants began the habituation task but were not included in the final sample because they became fussy during the task $(n=1)$, there was an experimental error $(n=4)$ or because their eye gaze was not visible on the camera for part of the task $(n=1)$. All infants participated in the toy-on-cloth condition.

\subsubsection{Materials and procedure}

3.1.2.1. Habituation task. Infants participated in the toy-on-cloth condition, which was identical to the toy-on-cloth condition of Study 1 (see also Fig. 1a).

3.1.2.2. Coding and reliability. The coding of infant eye gaze was identical to that of Study 1. A secondary observer, who was unaware of the condition that the infants participated in, coded the infants' eye gaze from videotape. The primary and secondary observers agreed on the ending of $88 \%$ of test trials. Bias analyses revealed that disagreements were randomly distributed $\left(X^{2}(\mathrm{df}=1)=0.42, P>0.52\right)$.

3.1.2.3. Action task. Infants took part in the action task either directly prior to (action first group) or directly after (habituation first group) participating in the habituation task.

Infants were tested in a room adjacent to the habituation room. Infants sat either on their parent's lap or in an adjustable high chair, behind a white table $(63.5 \mathrm{~cm} \times 51 \mathrm{~cm})$ of adjustable height. At the beginning of the task infants were given a yellow duck $(9 \mathrm{~cm} \times 9 \mathrm{~cm})$ to play with, until they appeared to be comfortable with the setting. At this point the experimenter took the yellow duck away from the infant, placed a red felt cloth $(43 \mathrm{~cm} \times 28 \mathrm{~cm})$ down in front of the infant, and placed the duck at the far end of the cloth (approximately $33 \mathrm{~cm}$ from the infant). Trials lasted until the infant pulled the cloth and retrieved the toy. If the infant did not retrieve the toy after $30 \mathrm{~s}$ had elapsed the experimenter picked up the toy and handed it to the infant. Each infant was given between 6 and 11 opportunities to solve the task based on their willingness to complete the task. The experimenter ceased administering trials when infants reached 11 trials, became fussy or bored, or failed to act on three or more trials.

During the task a number of different toys were used to maintain infants' interest (for example, an orange porcupine, a pink plastic cat, and a blue plastic bear). A gray corduroy 


\section{ARTCLLE IN PRESS}

cloth $(43 \mathrm{~cm} \times 28 \mathrm{~cm})$ was used in addition to the red felt cloth. The cloths were switched after even-numbered trials. Both the toys and the cloths used in the action task differed in color, size and texture from the habituation toys and cloths.

3.1.2.4. Coding. Willatts (1990) reports that infants as young as 6 months of age may pull the toy towards them in the course of playing with the cloth. Therefore, infants' responses were coded based on a scheme which incorporated elements of earlier coding schemes used by Bates et al. (1980) and Willatts (1999), which focused on the extent to which infants' actions were apparently a planful means to obtaining the outof-reach toy.

Planful strategies were scored according to the following criteria: the infant focused on the toy prior to reaching for the cloth, maintained visual fixation on the toy while pulling the cloth and grasped the toy once it came within reach (e.g. within $3 \mathrm{~s}$ ). Trials on which infants acted on the cloth or attempted to get the toy, but did not meet this criteria, were scored as unplanful. ${ }^{5}$ Trials on which the infant did not act were not coded (since it was not clear why the infant did not act). This coding was completed from videotape by an observer who was unaware of infants' habituation performance. An example of a planful strategy is depicted in Fig. 3.

3.1.2.5. Reliability. A second naïve coder coded $25 \%$ of the infants' action task trials from videotape. Agreement between the primary and secondary coder was $93 \%$.

\subsection{Results}

\subsubsection{Habituation task}

Fig. 2 summarizes infants' looking times for the habituation and test trial in each condition as a function of action task order.

3.2.1.1. Habituation. Overall infants averaged 7.6 habituation trials $(\mathrm{SE}=2.5)$. Infants in the action first group had an average of 7.8 habituation trials $(\mathrm{SE}=2.5)$ and infants in the habituation first group had an average of 7.4 habituation trials $(\mathrm{SE}=2.6)(t<1.0)$. Looking times on the last three habituation trials did not differ as a function of action task order $(M=21.7, \mathrm{SE}=2.5$, action first group; $M=18.5, \mathrm{SE}=2.6$, habituation first group; $t<1.0)$.

3.2.1.2. Test. Preliminary analyses revealed no reliable effects of the side to which the actor first reached, the toy on the actor's right, or the test trial type given first on infants' looking times. For this reason, infants' total looking times (across the three trials of each kind) were used in the subsequent analyses. There was a main effect of trial pair $(F(2,46)=8.33, P<0.001)$, indicating that looking declined across trial pairs, but no trial pair by trial type interaction.

\footnotetext{
${ }^{5}$ On a few trials ( $2 \%$ of all trials), the toy was inadvertently knocked off the table. In this case it was not possible for the child to grasp the toy. On these trials the infants' behavior was scored as planful if they continued to look for the toy after it was knocked from the table.
} 


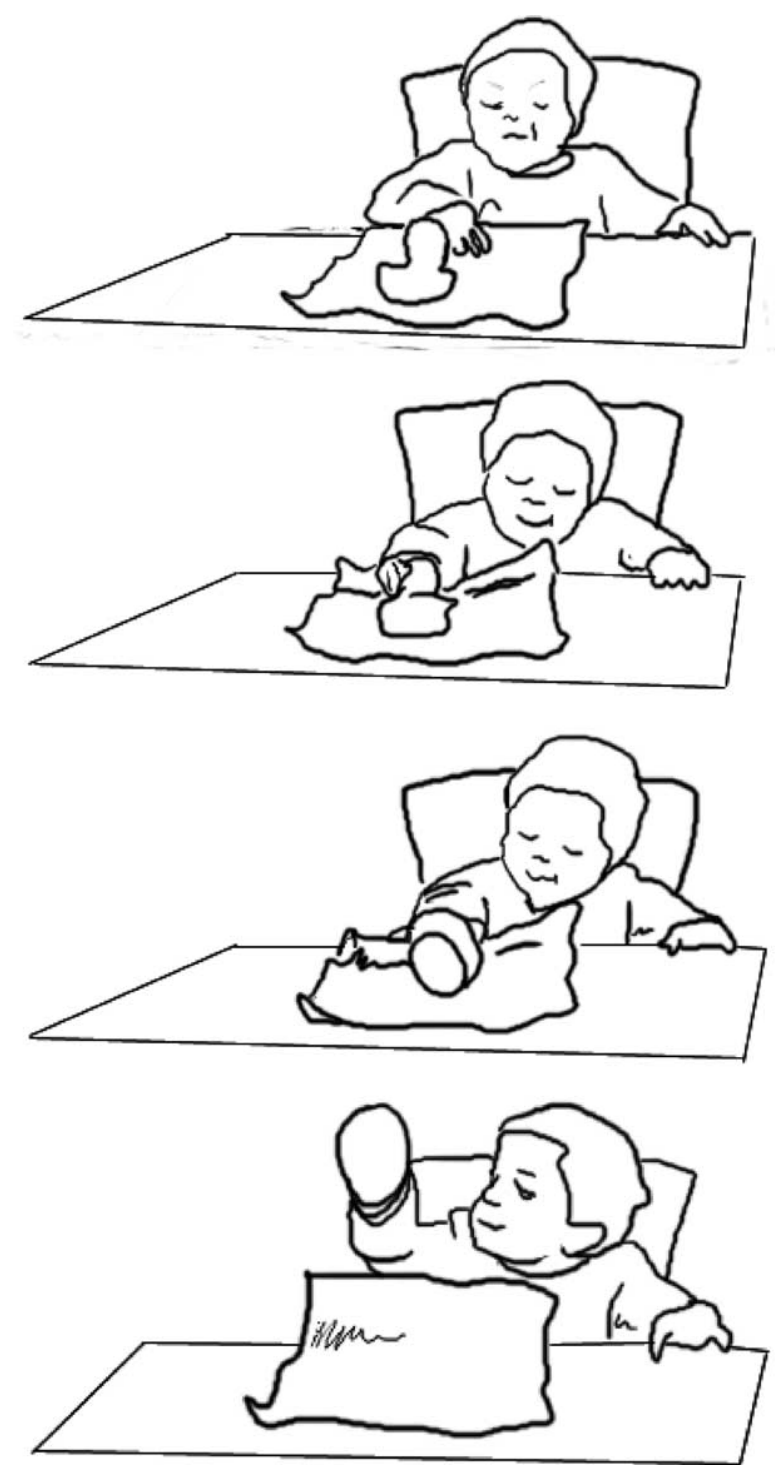

Fig. 3. A planful solution.

An ANOVA on infants' looking times to the test events with task order (action first vs. habituation first) as the between-subjects variable and trial type (new goal vs. new means event) as the within-subjects variable was performed. This analysis revealed no reliable effects $(F<1.0)$. These findings suggest that (a) as a group 10-month-old infants were not sensitive to the ultimate goal of the cloth-pulling sequence, and that (b) simply participating in the action task prior to viewing the habituation event did not influence infants' event interpretations. 


\section{ARTICLE TN PRESS}

Although looking time preferences and habituation rates were not different for infants who received the action task prior to the habituation task, versus those that received the tasks in the reverse order, it is possible that recovery from habituation may have differed as a function of action task order. Post-hoc analyses revealed that both groups of infants showed recovery to both types of test events (action first group: to new goal event $t(23)=2.29, P<0.03$, to new means event $t(23)=2.97 ; P<0.007$; habituation first group: to new goal event $t(23)=2.52, P<0.02$, to new means event $t(23)=3.11$, $P<0.005)$. That is, neither group of infants showed selective attention to a change in the actor's ultimate goal.

\subsubsection{Action task performance}

Because of the variability in the number of action task trials administered to infants we used as our dependent measure the percentage of trials on which infants produced planful strategies. On average, infants produced planful strategies on $59 \%$ of trials $(\mathrm{SE}=5 \%$; range $0-100 \%$ ). Planned comparisons revealed that the frequency of planful strategies did not vary as a function of action task order $(t(43)=1.25, P>0.22)$ : infants in the action first group produced planful strategies on $53 \%(\mathrm{SE}=8 \%)$ of trials and those in the habituation first group produced planful strategies on $65 \%(\mathrm{SE}=6 \%)$ of trials.

\subsubsection{The relation between action task and habituation task performance}

To investigate the extent to which individual performance on the action task and habituation task was related, performance on the two tasks were subject to a correlational analysis (see Fig. 4). For the habituation task, the magnitude and consistency of infants' preference for the new goal test event was used as a measure of infants' sensitivity to the actor's ultimate goal in the cloth-pulling sequence. This measure was obtained by averaging infants' preference for the new goal event (new goal-new means) across the three test trial pairs. For the action task, the frequency with which infants produced planful strategies was used as the dependent measure. This analysis revealed a significant correlation between action task and habituation task performance $(r=0.56, P<0.0001)$,

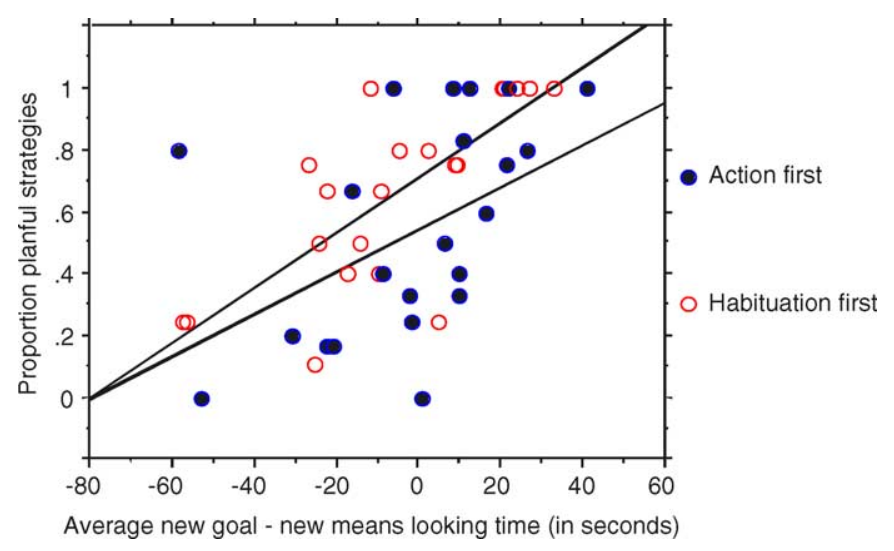

Fig. 4. Scatterplot of relation between habituation and action task performance, Study 2. 


\section{ARTICLE IN PRESS}

which remained significant even with age partialled out $(r=0.55, P<0.0001)$. This correlation was significant for both the action first group $(r=0.46, P<0.03$; agepartialled) and the habituation first group ( $r=0.71, P<0.0001$; age-partialled).

The findings from the correlational analysis suggest that sensitivity to the actor's ultimate goal in the habituation task is related to the frequency with which infants produce planful solutions in the action task. In a further analysis, infants were divided into two groups based on their performance on the action task. Infants were dubbed 'Planful infants' if they scored in the top 25\% on the action task (with respect to frequency of planful solutions; $n=12$ ). Planful infants produced planful strategies on $96 \%$ of trials $(\mathrm{SE}=3 \%$; range $83-100 \%)$. Infants who scored in the bottom $25 \%$ on the action task were dubbed 'Non-planful infants' $(n=12)$. Non-planful infants produced planful strategies on $10 \%$ of trials ( $\mathrm{SE}=4 \%$; range $0-20 \%$ ).

Fig. 2 depicts infants' looking time to the test events as a function of action task performance. To examine whether infants' interpretations of the habituation event differed as a function of action task performance, an ANOVA on infants' looking times to the test events with action task performance (planful vs. non-planful) as the between-subjects variable and trial type as the within-subjects variable revealed an action task by trial type interaction $(F(1,23)=27.61, P<0.0001)$ and no other reliable effects. Planned comparisons revealed that planful infants showed a significant preference for the new goal event $(t(11)=3.84, P<0.003)$, whereas non-planful infants showed a significant preference for the new means event $(t(11)=3.74, P<0.003)$. Non-parametric tests supported this pattern of findings. Whereas 10 of 12 planful infants showed a preference for the new goal event, 9 of 12 non-planful infants showed a preference for the new means event $\left(X^{2}(\mathrm{df}=1)=8.22, P<0.004\right)$. These findings suggest that whereas planful infants appreciated that the grasp of the cloth was directed toward the ultimate goal, non-planful infants appeared to interpret the grasp of the cloth as directed toward the cloth itself. ${ }^{6}$ The failure to find an overall effect of action task performance on looking times suggests that planful and non-planful infants did not differ in their attentiveness to the test events. In addition, planful and non-planful infants did not differ in terms of age $(t(22)=1.49$, $P>0.15)$, or looking time on the last three habituation trials $(t<1.0){ }^{7}$ These findings suggest that neither age nor attention to the habituation event can account for the differences between planful and non-planful infants with respect to their looking time preferences on the test events.

Infants' recovery from habituation to the test events was compared as a function of action task performance. Planned comparisons revealed that whereas planful infants significantly recovered attention to both the new goal event $(t(11)=4.41, P<0.001)$ and the new means event $(t(11)=2.41, P<0.04)$, non-planful infants only recovered attention to the new means event $(t(11)=3.14, P<0.009)$ and not to the new goal event

\footnotetext{
${ }^{6}$ It could also be argued, however, that non-planful infants were more sensitive to changes in the perceptual features of the event since the new means event featured a change in the cloth that the actor was grasping as well as a change in the location that she was reaching to.

${ }^{7}$ Planful infants actually took marginally more habituation trials to reach the habituation criterion than nonplanful infants $(t(23)=1.85, P<0.08)$. Note that this difference is in the opposite direction than would be predicted if information-processing capacity was thought to underlie the observed difference in habituation performance between planful and non-planful infants.
} 


\section{ARTICLEE TN PRESS}

$(t<1.0)$. These findings indicate that whereas planful infants were attentive to changes to the actor's ultimate goal and the intermediary that she acted on, non-planful infants were only attentive to changes in the intermediary on which the actor acted (e.g. the cloth that she grasped). These results differ from those of Study 1, in which 12-month-olds in the toy-on-cloth condition selectively recovered attention to the new goal event. One possibility is that even planful 10-month-olds are continuing to refine their action representations of simple means-end sequences.

\subsection{Discussion}

The findings from Study 2 suggest that there is an important transition in infants' ability to detect the intentional structure of a simple action sequence between 10 and 12 months of age. Whereas, in Study 1, 12-month-olds showed a novelty preference for events in which the relation between the actor and her ultimate goal was disrupted, 10-month-old infants, as a group, did not demonstrate this ability. In addition, being given the opportunity to perform the cloth-pulling sequence directly before viewing another person perform a similar sequence did not facilitate infants' interpretation of the sequence.

Further investigation of 10-month-olds' looking times revealed two distinct patterns. Infants who produced a high proportion of planful solutions on the action task showed a greater tendency to view the sequence as hierarchically organized. In addition, infants viewed the habituation event differently depending on how well they performed on the action task. Infants who were largely planful on the action task interpreted the actor's actions on the cloth as directed toward the toy. Infants who were largely non-planful on the action task showed the opposite pattern of looking: they focused on the relation between the actor and the cloth that she was grasping.

These findings highlight the fact that although infants as a group were insensitive to the goal-directed structure of the cloth-pulling task, there were individual differences in performance on the habituation task that could be accounted for once action task performance was considered. As such, the results rule out one interpretation of the habituation task performance of 10-month-olds: that infants of this age may fail to encode the actor's ultimate goal in the habituation task because the paradigm that we used was insensitive to their action knowledge. In addition, these findings provide some of the first empirical evidence to indicate a relation between the production and comprehension of the intentional structure of action in infancy. This topic will be considered in detail below.

\section{General discussion}

The ability to understand action as hierarchically organized figures centrally in adult event representations. It enables us to interpret novel or ambiguous actions, to predict or anticipate an actor's ultimate goal and to represent action at multiple levels, with various layers of embedding. Moreover, it may form the basis of our ability to understand action as motivated and guided by invisible internal states such as goals and intentions. The focus of this paper was on when and how infants develop the bare bones of this ability. 
The results from Study 1 converge with prior findings (Woodward \& Sommerville, 2000 ) in indicating that by 12 months of age infants understand actions as directed toward an overarching goal. After having seen an actor pull a cloth to retrieve a toy, infants interpreted the actor's subsequent actions on only the cloth as directed toward the toy (and not the cloth itself). In addition, 12-month-olds' understanding of this sequence appears to be governed by some of the same constraints as adults: infants applied their causal knowledge of the situation when making goal attributions.

The second aim of this paper was to examine the developmental time course for construing actions in a means-end sequence as directed toward an ultimate goal. Our findings identify $10-12$ months of age as an important transitional time in infants' developing ability to interpret intentional action. As such, our results are in general agreement with the widely held theoretical view that infants' understanding of intentional action undergoes critical developments at this age (e.g. Bates, Beningni, Bretherton, Camaioni, \& Volterra, 1979; Bretherton, 1991; Carpenter, Nagell, \& Tomasello, 1998; Meltzoff, 1999; Tomasello, 1999).

The third aim of this paper was to identify factors that may play a role in infants' developing ability to reason about the actions of others, by examining the potential relation between action production and understanding. Study 2 provides some of the first empirical evidence for the notion that action perception and production are closely linked (e.g. Baldwin, 1897; Mill, 1867), and that such a relation may serve as a powerful engine in development (Meltzoff \& Brooks, 2001; Tomasello, 1999; Woodward et al., 2001). Specifically, we found a relation between infants' ability to use goal-directed strategies to solve a cloth-pulling task and their ability to interpret the cloth-pulling behavior of others as directed toward an ultimate goal. In the following sections we reflect on the nature of infants' action representations, and speculate about the source of the relation between action processing and production in this context.

\subsection{What do 12-month-old infants know about plans and goals?}

Key to a mature understanding of intentions is the ability to recognize goals as mental states that are contextually malleable. Our data raise the question of whether infants possess these two aspects of goal understanding.

With respect to the first aspect, other investigators have argued that by 12 months of age infants attribute dispositional states and mental properties to animated figures that behave in apparently social ways (Kuhlmeier, Wynn, \& Bloom, 2003; Premack \& Premack, 1997). While our findings do not contradict this "rich interpretation" of infants' responses, it is important to point out that 12-month-olds' goal recognition as assessed in the current studies does not force us to the view that infants understand goals as mental entities per se. Instead, infants' understanding of the abstract nature of goals may be more primitive than that of adults (cf. Csibra et al., 2003). For instance, infants may understand goals or desires based on their accompanying tangible physical sensations (such as the yearning for an object; cf. Wellman, 1990). This understanding would be akin to an adult understanding of pain or hunger as internal non-representational states that are unique to individuals. Alternatively, infants may solely understand goals as residing in action, but appreciate that goals are not tied to a particular action (Meltzoff, 1995). 


\section{ARTICLEE TN PRESS}

With respect to the second aspect of goal understanding, our findings suggest that 12-month-olds' understanding of goals is contextually flexible in at least one way: infants take into account the causal constraints of the situation when linking actions to an ultimate goal. Adults use this information when creating their action interpretations and also incorporate other types of knowledge, including psychological information (e.g. about an actor's traits or tendencies), social/conventional information (e.g. about social customs or rituals) and their own beliefs about the anticipated consequences of action, when trying to determine an actor's goal.

The present findings do not allow us to decipher the extent of abstractness of infants' goal representations, or the range of constraints or information that infants can consider in forming their action construals. Nevertheless, the ability to view action as hierarchically organized and to integrate aspects of the observable context into one's action representations confers advantages over the restricted ability to process the relation between an actor and her proximal goals. This understanding provides the basic machinery by which infants may structure their event representations and, as such, may form the basis for infants' later understanding of goals and intentions as internal, unique, unobservable states that exist in their own and others' minds.

\subsection{The nature and source of infants' action understanding and production}

Our findings suggest that infants' ability to perform a cloth-pulling sequence in a planful manner is tightly connected to their ability to read beyond the proximal goal. Therefore, it is important to explore the nature and source of the relation between infants' action understanding and production.

\subsubsection{Generality}

The findings from Study 2 suggest that infants who performed well on the action task did not differ from those who performed poorly in terms of at least two variables: age (a proxy for developmental level) and habituation rate (a proxy for information-processing capacity). These findings suggest that it is unlikely that a third variable (such as general intelligence, information-processing capacity, or overall maturational level) can account for the relation between action perception and production. However, because the role of these factors was not directly assessed in current study, additional empirical work is needed to rule such an interpretation (e.g. exploring the effect of training on means-end sequences on infants' interpretation of such sequences).

Although domain-general abilities no doubt contribute to infants' ability to build hierarchical action representations and to their ability to perform action sequences in their own behavior, we believe that such abilities cannot entirely account for the relation between action production and perception documented in Study 2. The ability to perform means-end sequences varies as a function of a variety of task-specific variables (Bates et al., 1980) and there is decalage in infants' ability to perform similar means-end tasks (Piaget, 1953), despite the fact that tasks may not differ from one another appreciably with respect to various domain-general factors (such as the amount of inhibition required to solve the task). Second, recent work suggests that means-end performance can be improved via modeling (e.g. Menard \& Aguiar, 2002), which is at odds with 
the interpretation that means-end performance is exclusively governed by developing domain-general capacities. Finally, infants in the current study demonstrated subtle "priming" effects similar to those documented in the adult motor priming literature (e.g. Castiello, Lusher, Mari, Edwards, \& Humphreys, 2002). Infants who received the action task after watching the habituation task performed a (non-significantly) higher proportion of planful strategies (65\%) than did infants who received the action task first (53\%). Similar priming effects have recently been documented in 9- and 11-month-old infants (Hauf, Prinz, \& Aschersleben, 2003). Such priming effects are thought to rely on shared coding of observed and executed actions, and cannot be fully explained by a domaingeneral account.

Instead, we speculate that our findings may reflect the operation of an observation/execution matching system, similar or identical to the one that has been documented in nonhuman primates and adult humans. Such a system may indeed be present in infants beginning at 10 months of age if not earlier (see also Meltzoff, 2002a). In the proceeding section, we consider processes that may operate within such a system and the direction in which shared representations may be first established (e.g. self to other vs. other to self).

\subsubsection{Processes involved in self-other mapping}

If infants' developing ability to produce action sequences leads them to understand observed action sequences this could occur via several processes. First, infants' ability to produce particular actions may give them more exemplars of an action by which the intentional structure may be pulled out. Recently, some authors have argued that structural information facilitating goal identification may be present when observing goal-directed behavior. For instance, Baldwin and colleagues (Baird \& Baldwin, 2001; Baldwin, Baird, Saylor, \& Clark, 2001) suggest that there are cues associated with the completion of object-directed goals or intentions (such as bodily orientation, movement toward, contact with, and release of, objects) that infants become sensitive to and use to make goalrelevant segmentations. Similarly, Jovanovic et al. (under review) suggest that action outcomes play a crucial role in the identification of action goals. As such, infants developing expertise at performing the cloth-pulling sequence may allow them to observe more exemplars specifying the goal structure of the action. According to this account, observation of action exemplars in other people's behavior would be just as effective in enabling infants to understand the actions of others.

Second, increasing expertise in the production of a given action sequence may lead to changes in infants' allocation of attention, such that other aspects of the task can be attended to. There is a rich body of work suggesting that changing action capabilities drive changes in visual attention to events, objects and surfaces (e.g. Gibson, 1988; Gibson \& Pick, 2000). For instance, Needham, Barrett, and Peterman (2002) demonstrated that providing pre-reaching infants with experience apprehending objects increased their visual exploration of objects. Developing action capabilities may lead to changes not only in the amount of visual attention that infants allocate to objects and events, but to the nature of this attention: infants who are highly skilled at object handling attend more to intermodal information than less skilled infants (Eppler, 1995). Furthermore, work with adults provides direct evidence that gaining expertise at skill acquisition is associated with a change in attention during skilled performance (e.g. Wulf and Prinz, 2001). 


\section{ARTICLE TN PRESS}

One possibility, then, is that the developing ability to perform the cloth-pulling task may change infants' attention to the goal-related structure of the task.

A third possibility is that infants' ability to reflect upon their own goals in a similar context may provide them with information about the actions of others that could not be obtained simply from watching someone act (e.g. Meltzoff, 2002b). Infants may understand the actions of the actor based on a simulation of those actions. For instance, when the infant watches the habituation event, she may assume that when the actor pulls the cloth the actor 'has the goal of' getting the toy, based on the fact that when she (the infant) pulls the cloth she (the infant) intends to get the toy. This accounts requires (a) that the infant have some awareness of her goals in acting and (b) that the infant has access to that prior intention in the habituation task. While such an account may seem unlikely given work with adults that suggests a general lack of awareness of the mental states that accompany their actions (e.g. Nisbett \& Wilson, 1977), it is possible that low-level goals or intentions may represent particularly salient mental states that are accessible to both adults and infants. Alternately, it is possible that the informational value of agentive experience does not require that the agent be explicitly aware of his or her intentional states.

\subsubsection{Direction of the relation}

It is also possible that the direction of the relation flows from other to self. Infants may learn about the intentional structure of the actions of others by observing them, and then come to apply this knowledge in their own action production. In one study, Provasi, Dubon, and Bloch (2001) found that 12-month-old infants given the opportunity to watch an adult solve a means-end task were more successful at subsequently generating the solution than infants merely given the opportunity to play with the apparatus themselves. Using an imitation paradigm, Hauf et al. (in press) found that infants perform actions that had been demonstrated to produce interesting effects more frequently and more quickly than other actions. Although future work must be done to equate observation experience with practice experience, these findings suggest infants can acquire information about how to reproduce the goal of an action sequence by observing another person act.

It seems likely that the link between action understanding and production is bi-directional, particularly by late infancy. Meltzoff (2002b) proposed what he calls the "Like Me" hypothesis, which embodies this idea. According to this hypothesis, infants can use self-experience to interpret the actions of others, and reciprocally can use the actions of others as models for the self. This is thought to play a major role in infancy: "That young infants can interpret the acts of others in terms of their own acts and experiences provide them with enormous leverage and an engine for development" (Meltzoff, 2002b, p. 22). Infant imitation is highlighted as the prime manifestation of the close coupling between action perception and production, but the "Like Me" hypothesis has also been applied to joint attention and understanding others' intentions (Meltzoff \& Brooks, 2001; Meltzoff \& Moore, 1995, 1997). This hypothesis is compatible with the current finding that infants' means-ends production is tightly coupled with their understanding of meansends behavior in others. Moreover, taken as a whole, this emerging body of infancy work fits well with the neuroscience findings of shared neural networks for both observed and executed actions (e.g. Meltzoff \& Decety, 2003). 


\subsection{Implications for developmental theories}

The results of the current study have both general and specific implications for developmental theories. First, in keeping with Piaget (1953), we demonstrate the potential importance of action experience for representational understanding. Our findings also demonstrate the utility of specific information gleaned from the production of particular actions for understanding those actions. As such, our results provide important information about the manner in which early experience may contribute to representational understanding.

By assessing action representations across modalities, our results have implications for the accessibility of knowledge measured by habituation tasks to other response systems. To date, very few studies have examined the relation between habituation performance and "real world" behavior (but see Eppler, 1995; Needham et al., 2002). The findings from the present study suggest that the results of looking time studies extend beyond the habituation booth. As such, they raise the possibility that previously observed dissociations between looking time data and action task performance may depend as much on task demands as they do on the response modality used to gauge infants' response (cf. Munakata, 2001).

Furthermore, the findings from the current study have specific implications for theories and proposals of early intentional understanding. Some investigators have claimed that viewing action as intentional is an innate endowment that enables infants to make abstract judgments based on the presence of a single or small set of cues signaling intentionality (e.g. Premack, 1990). However, our findings that infants' action representations (a) change with development and (b) are intimately tied to infants' own ability to perform similar actions, suggest that the ability to view action as hierarchically organized is best characterized as a developmental achievement. While our findings do not rule out the possibility that such perceptual modules exist, they strongly suggest that other factors, chief among them experience as an agent, structure infants' developing action knowledge. As such, our results align with a constructivist perspective on early intentional understanding (e.g. Baird \& Baldwin, 2001; Baldwin et al., 2001; Carpenter et al., 1998; Gopnik \& Meltzoff, 1997; Tomasello, 1999), and contribute to this literature by adding new information regarding the nature of infants' developing action representations in the first year of life and the types of experiences that contribute to these changes.

Recently, Csibra et al. (2003) have proposed an alternative interpretation of our earlier findings (Woodward \& Sommerville, 2000) in terms of their proposed teleological system that could, in principle, be extended to the current findings. Under this account infants analyze object motion in terms of a teleological schema that includes the observed behavior, potential future states of affairs, and the physical constraints of the situation (e.g. Csibra et al., 2003, 1999; Gergely et al., 1995; Gergely \& Csibra, 1997). Movement is interpreted as goal-directed if it meets well-formedness conditions, specified by the rationality principle (an object taking the most efficient route to the target object). From this perspective, one interpretation of our findings is that infants demonstrated longer looking on the new goal trials because the familiar action was no longer a rational means to attaining the goal of the sequence (see Csibra et al., 2003). In contrast, infants may not have encoded the clothpulling act as goal-directed in the control condition because it did not meet the rationality criteria: pulling the cloth was not interpreted as an efficient means to attain the toy. 


\section{ARTICLEE TN PRESS}

Our account of action understanding differs from Csibra and colleagues in a number of ways. At a general level, we believe that studying infants' interpretation of human goaldirected action (as opposed to motion trajectories of inanimate objects) is the proper domain for tapping infants' action representations. Whereas humans perform action end states that roughly correspond to mental goals, inanimate objects do not. Furthermore, recent neuroimaging work suggests that object motion and human action are processed differently, with the motor system selectively activated during the perception of possible human actions (Stevens, Folupt, Shiffrar, \& Decety, 2000). Although this work cannot inform us about the starting state of human action and object motion processing, it at least raises the possibility that processing across these domains is not commensurate.

In addition, our view differs from Csibra and Gergely's account in terms of the output of infants' action representations. Csibra and Gergely's account focuses on the necessary criteria for a given act to be evaluated as goal-directed or not. In contrast, our view seeks to assess when and how infants identify the ultimate goal of the sequence within the stream of behavior. When infants fail to demonstrate longer looking on the new goal trials we believe that it may be because they misidentify the goal of the sequence as the cloth rather than the toy. As such, this "error" reflects a misidentification of the actor's goal, rather than a miscategorization of the act itself. Indeed, the findings from 10-month-olds who were non-planful on the action task support this speculation: these infants demonstrated significantly longer looking on the new means trials indicating that they may have (mis)interpreted the actor's goal as the cloth rather than the toy.

\section{Conclusion}

In closing, the findings from the present study add to the growing body of literature investigating developments in action representations during the first several years of life. Our findings indicate that by 12 months of age infants create action representations that are centered around an overarching action goal. Moreover, infants' action representations are informed by their causal knowledge. These developments represent an important advance in infants' action processing, as viewing action as hierarchically organized and using topdown knowledge to organize action representations is a key component of older children's and adults' event knowledge. Our findings further suggest that 10-12 months of age is transitional period with respect to action understanding and point to a mechanism by which infants may come to understand the actions of others: by observing and executing particular actions in their own behavior. As such, our results are consistent with research indicating a common basis for the production and perception of actions and suggest that action observation and execution rely on a shared code starting in infancy.

\section{Acknowledgements}

The studies reported in this paper were completed in partial fulfillment of a Doctor of Philosophy degree to the first author. We thank Elizabeth Perkowski and the staff at the Center for Infant Studies at the University of Chicago for their help in conducting 
the studies, and the parents and infants who graciously devoted their time and energy to participate in this research. We are grateful to Bennett Bertenthal, Susan Goldin-Meadow, and Janellen Huttenlocher for input in the early stages of this project and to Cristina Atance, Andrew Meltzoff and Betty Repacholi and four anonymous reviewers for their comments on a prior version of this article. This work was supported by a McCormick Tribune Dissertation Fellowship to the first author and by an NIH grant (R29-HD35707) to the second author.

\section{References}

Baird, J., \& Baldwin, D. A. (2001). Making sense of human behavior: Action parsing and intentional inference. In B. F. Malle, L. J. Moses, \& D. A. Baldwin (Eds.), Intentions and intentionality: Foundations of social cognition. Cambridge, MA: MIT Press.

Baldwin, D. A., Baird, J. A., Saylor, M. M., \& Clark, M. A. (2001). Infants parse dynamic action. Child Development, 72, 708-717.

Baldwin, J. M. (1897). Social and ethical interpretations in mental development: A study in social psychology. New York: Macmillan.

Bartsch, K., \& Wellman, H. M. (1995). Children talk about the mind. Oxford: Oxford University Press.

Bates, E., Beningni, L., Bretherton, I., Camaioni, L., \& Volterra, V. (1979). The emergence of symbols: Cognition and communication in infancy. New York: Academic Press.

Bates, E., Carlson-Luden, V., \& Bretherton, I. (1980). Perceptual aspects of tool using in infancy. Infant Behavior and Development, 3, 127-140.

Blakemore, S. J., \& Decety, J. (2001). From the perception of action to the understanding of intention. Nature Reviews Neuroscience, 2, 561-567.

Bretherton, I. (1991). Intentional communication and the development of an understanding of mind. In D. Frye, \& C. Moore (Eds.), Children's theories of mind: Mental states and social understanding (pp. 49-75). Hillsdale, NJ: Lawrence Erlbaum Associates.

Carpenter, M., Akhtar, N., \& Tomasello, M. (1998). Fourteen- through 18-month-old infants differentially imitate intentional and accidental actions. Infant Behavior and Development, 21, 315-330.

Carpenter, M., Nagell, K., \& Tomasello, M. (1998). Social cognition, joint attention, and communicative competence from 9 to 15 months of age. Monographs of the Society for Research in Child Development, 63, $1-176$.

Carpenter, M. N., Call, J. P., \& Tomasello, M. (2002). Understanding prior intentions enables 2-year-olds to imitatively learn a complex task. Child Development, 75, 1431-1441.

Castiello, U., Lusher, D., Mari, M., Edwards, M., \& Humphreys, G. W. (2002). Observing a human or a robotic hand grasping an object: Differential motor priming effects. In W. Prinz, \& B. Hommel (Eds.), Common mechanisms in perception and action: Attention and performance XIX (pp. 315-333). Oxford: Oxford University Press.

Chen, Z., \& Siegler, R. S. (2000). Across the great divide: Bridging the gap between understanding of toddlers' and older children's thinking. Monographs of the Society for Research in Child Development, 65, 1-108.

Cohen, L. B. (1998). An information-processing approach to infant perception and cognition. In F. Simion, \& G. Butterworth (Eds.), The development of sensory, motor and cognitive capacities in early infancy: From perception to cognition (pp. 277-300). Hove, England: Psychology Press.

Cohen, L. B., Chaput, H. H., \& Cashon, C. H. (2002). A constructivist model of infant cognition. Cognitive Development, 17, 1323-1343.

Cooley, C. H. (1902). Human nature and the social order. New York: Scribner's.

Csibra, G., Biro, S., Koos, O., \& Gergely, G. (2003). One-year-old infants use teleological representations of actions productively. Cognitive Science, 27, 111-133.

Csibra, G., Gergeley, G., Biro, S., Koos, O., \& Brockbank, M. (1999). Goal attribution without agency cues: The perception of "pure reason" in infancy. Cognition, 72, 237-267. 


\section{ARTICLE TN PRESS}

Diamond, A. (1985). Development of the ability to use recall to guide action, as indicated by infants' performance on AB. Child Development, 55, 868-883.

Diamond, A. (1991). Neuropsychological insights into the meaning of object concept development. In S. Carey, \& R. Gelman (Eds.), The epigenesis of mind: Essays on biology and cognition. The Jean Piaget Symposium series (pp. 67-110). Hillsdale, NJ: Lawrence Erlbaum Associates.

Eppler, M. A. (1995). Development of manipulatory skills and the deployment of attention. Infant Behavior and Development, 18, 391-405.

Gallese, V., \& Goldman, A. (1998). Mirror neurons and the simulation theory of mind reading. Trends in Cognitive Science, 12, 493-501.

Gergely, G., \& Csibra, G. (1997). Teleological reasoning in infancy: The infant's naive theory of rational action. A reply to Premack and Premack. Cognition, 63, 227-233.

Gergely, G., Bekkering, H., \& Kiraly, I. (2002). Rational imitation in preverbal infants. Nature, 415, 755.

Gergely, G., Nasady, Z., Csibra, G., \& Biro, S. (1995). Taking the intentional stance at 12 months of age. Cognition, 56, 165-193.

Gibson, E. J. (1988). Exploratory behavior in the development of perceiving, acting, and the acquiring of knowledge. Annual Review of Psychology, 39, 1-41.

Gibson, E. J., \& Pick, A. D. (2000). An ecological approach to perceptual learning and development. Oxford: Oxford University Press.

Goldman, A. (1989). Interpretation psychologized. Mind and Language, 4, 161-185.

Gopnik, A., \& Meltzoff, A. N. (1997). Words, thoughts and theories. Cambridge, MA: MIT Press.

Gordon, R. M. (1986). Folk psychology as simulation. Mind and Language, 1, 158-171.

Harris, P. (1989). Children and emotion. Oxford: Blackwell.

Hauf, P. Elsner, B., \& Aschersleben, G (in press). The role of action effects in infant's action control. Psychological Research.

Hauf, P., Prinz, W., \& Aschersleben, G (2003). "You-like-me" or "Me-like you"cognition and off-line simulation: Two ways of understanding the simulation approach. Mind and Language, 13, 477-498

Heineman-Peiper, J., \& Woodward, A. (2003). Understanding infants' understanding of intention: Two problems of interpretation. Consciousness \& Cognition, 12, 770-772.

Hood, B., Cole-Davies, V., \& Dias, M. (2003). Looking and search measures of object knowledge in preschool children. Developmental Psychology, 39, 61-70.

Jovanovic, B., Kiraly, I., Elsner, B., Gergely, G., Prinz, W., \& Aschersleben, G (under review). The role of effects for infants' perception of action goals.

Kiraly, I., Jovanovic, B., Prinz, W., Aschersleben, G., \& Gergely, G. (2003). The early origins of goal attribution in infancy. Consciousness and Cognition, 12, 752-769.

Kuhlmeier, V., Wynn, K., \& Bloom, P. (2003). Attribution of dispositional states by 12-month-olds. Psychological Science, 14, 402-408.

Meltzoff, A. N. (1995). Understanding the intentions of others: Re-enactment of intended acts by 18-month-old children. Developmental Psychology, 31, 838-850.

Meltzoff, A. N. (1999). Origins of theory of mind, cognition and communication. Journal of Communication Disorders, 32, 251-269.

Meltzoff, A. N. (2002a). Elements of a developmental theory of imitation. In A. N. Meltzoff, \& W. Prinz (Eds.), The imitative mind: Development, evolution, and brain bases (pp. 19-41). Cambridge: Cambridge University Press.

Meltzoff, A. N. (2002b). Imitation as a mechanism of social cognition: Origins of empathy, theory of mind, and the representation of action. In U. Goswami (Ed.), Blackwell handbook of childhood cognitive development. Blackwell handbooks of developmental psychology (pp. 6-25). Malden, MA: Blackwell.

Meltzoff, A. N., \& Brooks, R. (2001). "Like me" as a building block for understanding other minds: Bodily acts, attention and intention. In B. F. Malle, L. J. Moses, \& D. A. Baldwin (Eds.), Intentions and intentionality: Foundations of social cognition (pp. 125-148). Cambridge, MA: MIT Press.

Meltzoff, A. N., \& Decety, J. (2003). What imitation tells us about social cognition: A rapprochement between developmental psychology and cognitive neuroscience. Philosophical Transactions of Royal Society of London B, 358, 491-500. 


\section{ARTICLE TN PRESS}

Meltzoff, A. N., \& Moore, M. K. (1995). Infants' understanding of people and things: From body imitation to folk psychology. In J. Bermúdez, \& N. Eilan (Eds.), The body and the self (pp. 43-69). Cambridge, MA: MIT Press.

Meltzoff, A. N., \& Moore, M. K. (1997). Explaining facial imitation: A theoretical model. Early Development and Parenting, 6, 179-192.

Menard, K. R., \& Aguiar, A (2002, April). Facilitating infants' reasoning about means-end support and search problems. Paper presented at the biennial meeting of the International Society for Infant Studies, Toronto, Ont.

Mill, J. S. (1867). Examination of Sir William Hamilton's philosophy. London: Longmans.

Munakata, Y. (2001). Graded representations in behavioral dissociations. Trends in Cognitive Sciences, 5, 309-315.

Munakata, Y., McClelland, J. L., Johnson, M. H., \& Siegler, R. S. (1997). Rethinking infant knowledge: Toward an adaptive process account of successes and failures in object permanence tasks. Psychological Review, 104, $686-713$.

Needham, A., Barrett, T., \& Peterman, K. (2002). A pick me up for infants' exploratory skills: Early simulated experiences reaching for objects using 'sticky' mittens enhances young infants' object exploration skills. Infant Behavior and Development, 25, 279-295.

Newell, A., \& Simon, H. A. (1972). Human problem solving. Englewood Cliffs, NJ: Prentice Hall.

Newtson, D., \& Engquist, G. (1976). The perceptual organization of ongoing behavior. Journal of Experimental Social Psychology, 12, 436-450.

Nisbett, R. E., \& Wilson, T. D. (1977). On telling more than we can know: Verbal reports on mental processes. Psychological Review, 84, 231-259.

Piaget, J. (1953). The origins of intelligence in the child. London: Routledge \& Kegan Paul.

Pinto, J (1994). MacXHab (Version 3.1). Stanford, CA.

Premack, D. (1990). The infants' theory of self-propelled objects. In D. Frye, \& C. Moore (Eds.), Children's theories of mind: Mental states and social understanding (pp. 303-325). Hillsdale, NJ: Lawrence Erlbaum Associates.

Premack, D., \& Premack, A. J. (1997). Infants attribute value + /- to the goal-directed actions of self-propelled objects. Journal of Cognitive Neuroscience, 9, 848-856.

Provasi, J., Dubon, C. D., \& Bloch, H. (2001). Do 9- and 12-month-olds learn means-ends relation by observing? Infant Behavior and Development, 24, 195-213.

Rizzolatti, G., Fadiga, L., \& Gallese, V. (2001). Neurophysiological mechanisms underlying the understanding and imitation of action. Nature Reviews Neuroscience, 2, 661-670.

Schank, R., \& Abelson, R. (1977). Scripts plans goals and understanding: An inquiry into human knowledge structures. Hillsdale, NJ: Lawrence Erlbaum Associates.

Searle, J. R. (1983). Intentionality: An essay in the philosophy of mind. Cambridge: Cambridge University Press.

Slackman, E. A., Hudson, J. A., \& Fivush, R. (1986). Actions, actors, link and goals: The structure of children's event representations. In K. Nelson (Ed.), Event knowledge: Structure and function in development (pp. 47-69). Hillsdale, NJ: Erlbaum.

Stevens, J., Fonlupt, P., Shiffrar, M., \& Decety, J. (2000). New aspects of motion perception: Selective neural encoding for apparent human movements. NeuroReport, 11, 109-115.

Tomasello, M. (1999). Having intentions, understanding intentions and understanding communicative intentions. In P. D. Zelazo, J. W. Astington, \& D. R. Olson (Eds.), Developing theories of intention: Social understanding and self-control (pp. 63-75). Mahwah, NJ: Lawrence Erlbaum Associates.

Travis, L. L. (1997). Goal-based organization of event memory in toddlers. In P. W. van den Broek, P. J. Bauer, \& T. Bovig (Eds.), Developmental spans in event comprehension and representation: Bridging fictional and actual events (pp. 111-138). Mahwah, NJ: Erlbaum.

Uzgiris, I. C., \& Hunt, J. M. (1975). Assessment in infancy: Ordinal scales of psychological development. Chicago, IL: University of Illinois Press.

Wellman, H. M. (1990). The child's theory of mind. Cambridge, MA: MIT Press.

Wellman, H. M., \& Phillips, A. T. (2001). Developing intentional understandings. In B. F. Malle, L. J. Moses, \& D. A. Baldwin (Eds.), Intentions and intentionality: Foundations of social cognition (pp. 125-148). Cambridge, MA: MIT Press. 


\section{ARTICLE TN PRESS}

J.A. Sommerville, A.L. Woodward / Cognition xx (2004) xxx-xxx

Wenner, J. A., \& Bauer, P. J. (1999). Bringing order to the arbitrary: One- to two-year olds' recall of event sequences. Infant Behavior and Development, 22, 585-590.

Willatts, P. (1990). Development of problem-solving strategies in infancy. In D. F. Bjorklund (Ed.), Children's strategies: Contemporary views of cognitive development (pp. 23-66). Hillsdale, NJ: Lawrence Erlbaum Associates.

Willatts, P. (1999). Development of means-end behavior in young infants: Pulling a support to retrieve a distant object. Developmental Psychology, 35, 651-667.

Woodward, A. L. (1998). Infants selectively encode the goal object of an actor's reach. Cognition, 69, 1-34.

Woodward, A. L. (1999). Infants' ability to distinguish between purposeful and non-purposeful behaviors. Infant Behavior and Development, 22, 145-160.

Woodward, A. L. (2003). Infants' developing understanding of the link between looker and object. Developmental Science.

Woodward, A. L., \& Sommerville, J. A. (2000). Twelve-month-old infants interpret action in context. Psychological Science, 11, 73-77.

Woodward, A. L., Sommerville, J. A., \& Guajardo, J. J. (2001). How infants make sense of intentional action. In B. F. Malle, L. J. Moses, \& D. A. Baldwin (Eds.), Intentions and intentionality: Foundations of social cognition (pp. 149-169). Cambridge, MA: MIT Press.

Wulf, G., \& Prinz, W. (2001). Directing attention to movement effects enhances learning: A review. Psychonomic Bulletin and Review, 8, 648-660.

Zacks, J., Braver, T. S., Sheridan, M. A., Donaldson, D. I., Snyder, A. Z., Ollinger, J. M., Buckner, R. L., \& Raichle, M. E. (2001). Human brain activity time-locked to perceptual event boundaries. Nature Neuroscience, 4, 651-655.

Zacks, J. M., \& Tversky, B. (2001). Event structure in perception and conception. Psychological Bulletin, 127, $3-21$.

Zacks, J. M., Tversky, B., \& Iyer, G. (2001). Perceiving, remembering, and communicating in events. Journal of Experimental Psychology: General, 130, 29-58. 